\title{
Le traitement actuel de l'impuissance chez le paraplégique
}

\author{
Albert LERICHE \\ Hospices Civils de Lyon, Hôpital Henry Gabrielle, Unité d'Urologie, \\ Route de Vourles, BP 57, 69565 Saint-Genis Laval Cedex
}

\section{RESUME}

L'auteur résume ses 25 années d'expérience du traitement de l'impuissance du paraplégique pour expliquer l'aboutissement actuel à l'utilisation quasi constante des auto-injections de drogues vaso-actives. La rééducation sexuelle, basée sur le niveau neurologique, donc sur la physiopathologie, a disparu depuis 10 ans, comme la rééducation urinaire supplantée par l'autosondage. Cependant, il reste des principes d'éducation du paraplégique et du couple, tant dans la communication que dans la technique. La recherche de l'orgasme du paraplégique est devenue prioritaire, puisque l'érection est obtenue facilement pharmacologiquement. La place des médications vaso-actives est rappelée. La conduite à tenir est définie, un peu différente pour le paraplégique traumatique complet que pour le paraplégique incomplet (queue de cheval, spina, SEP), où la psychologie est différente, les troubles de la sensibilité communs à tous ne devant pas faire oublier qu'il existe toujours des zones érogènes réflexes, qu'il faut rechercher.

Mots clés : Paraplégique, impuissance, drogues vaso-actives.

\section{INTRODUCTION}

Il y a 15 ans, nous écrivions [4] que la rééducation sexuelle du paraplégique était une nécessité au même titre que la rééducation urinaire, pendant la même période, puisqu'elle avait fait passer en 5 ans le pourcentage de paraplégiques sans vie sexuelle de $50 \%$ à $20 \%$. Les traitements pharmacologiques, difficiles à manier ne permettaient une amélioration de l'érection que dans $20 \%$ des cas seulement.

L'apparition des injections intra-caverneuses de drogues vasoactives en 1982 [11] et en 1983 [1] a fait disparaître la rééducation sexuelle de l'homme paraplégique, en même temps que l'autosondage a fait disparaître la rééducation urinaire. Il existe maintenant dans le génito-urinaire un certain mépris de la physiopathologie qui était la base de la rééducation. Les vessies centrales, réflexes, automatiques sont transformées en vessies périphériques acontractiles, incapables de se vider, mais aussi parfaitement continentes. L'homme paraplégique, à l'exemple de la femme, s'autosonde. Le social a primé sur la physiologie. En même temps, il a récupéré des érections de bonne qualité, quel que soit son niveau neurologique, grâce aux auto-injections intracaverneuses. Il est exceptionnel actuellement de ne pas trouver une médication vasoactive adéquate, si bien que l'on peut quasiment affirmer obtenir une érection chez un paraplégique à quelques exceptions près [6] .

Notre recul est, comme pour l'autosondage, d'un peu plus de 10 ans. Les incidents surtout marqués par des priapismes récidivants, n'ont pas eu de conséquence facheu- 
se, pas plus que l'autosondage n'a entraîné de fausses-routes, rétrécissements uréthraux ou infections urinaires de traitement difficile. Ces complications, qui existent certes, ont toujours été de traitement facile. Cela est vraisembablement dû à une meilleure information du paraplégique par rapport au patient classique. Le paraplégique gère mieux lui-même ses propres complications. Le paraplégique est capable de se ponctionner lui-même un priapisme, de refaire cette ponction à chaque récidive, alors qu'il est rare qu'un patient ayant fait un priapisme, poursuive les auto-injections tant il a peur de récidiver.

Il n'empêche que nous sommes en train d'assister à un nouveau changement de moeurs. En même temps que certains patients se posent la question de leur mode mictionnel et de l'idée de ne pas poursuivre l'autosondage toute leur vie, certains découvrent la possibilité d'avoir des érections de manière différente, réflexe, mais aussi psychogène, la possibilité d'utiliser le garrot. Bien sûr, le fait d'avoir réalisé des bilans systématiques d'urodynamique, manométrie anorectale, électromyographie, pléthysmographie nocturne, sous stimulation visuelle, après injection intra-caverneuse etc... [3] a évidemment éveillé leur curiosité mais leur a fait aussi découvrir des potentialités qu'ils n'imaginaient pas.

\section{RAPPEL ANATOMOPHYSIOLOGIQUE DE L'ERECTION APRES LESION NEU- ROLOGIQUE (FIGURE 1)}

Il existe un centre médullaire de l'érection, au niveau du cône terminal (S2, S3, S4). Ce centre se calque sur le centre mictionnel principal, mais avec une prépondérance pour $\mathrm{S} 4$, alors que le détrusor correspond plutôt à S3. L'innervation est également calquée sur celle du système vésico-sphinctérien.

- Le système parasympathique avec les nerfs érecteurs assure le remplissage veineux , donc l'érection molle.
- Le système somatique avec les nerfs honteux internes assure la rigidité et une partie du remplissage.

- Le système sympathique est plutôt inhibiteur et n'est pas en relation directe avec le cône terminal.

La fonction érectile va donc être, chez l'homme spinal, similaire à la fonction vésicale.

Quand le cône terminal reste fonctionnel, la miction et les érections peuvent se produire dans des conditions proches de la normale. Comme la miction, l'érection se déclenche et s'entretient de façon réflexe, mécanique.

Aussi, toute atteinte médullaire siégeant :

- Au-dessus de D12 vertébral (à l'aplomb de S2-S3 médullaire) permet une érection indépendante du contrôle de la volonté et déclenchée de manière réflexe.

- Au-dessous de D12, risque d'interrompre l'arc réflexe.

\section{LES SIGNES CLINIQUES DES TROUBLES SEXXULS DU PARAPLEGIQUE}

Il s'agit là de constatations cliniques pratiquées par divers auteurs en dehors de toute rééducation sexuelle. Ces constatations ne confirment pas toujours les hypothèses physiopathologiques. Ainsi, on ne retrouve pas $100 \%$ d'érection dans les sections complètes qui respectent le centre $\mathrm{S} 2, \mathrm{~S} 3, \mathrm{~S} 4$ (au-dessus de D12 vertébral) avec automatisme médullaire rétabli.

L'érection n'est présente dans ces lésions médullaires complètes et spasmodiques que dans 80 à $93 \%$ des cas. Il faut, en fait, tenir compte de l'équilibre mictionnel, de l'intégrité complète de l'appareil vésico-sphinctérien et même de l'élément psychologique (malgré le caractère complet de la section).

Cette différence entre clinique et physiopathologie s'accentue lorsqu'on recherche l'éjaculation. Celle-ci n'est possible que dans 30 à $50 \%$ des lésions incomplètes, spasmodiques et dans $70 \%$ des lésions incomplètes flasques. 


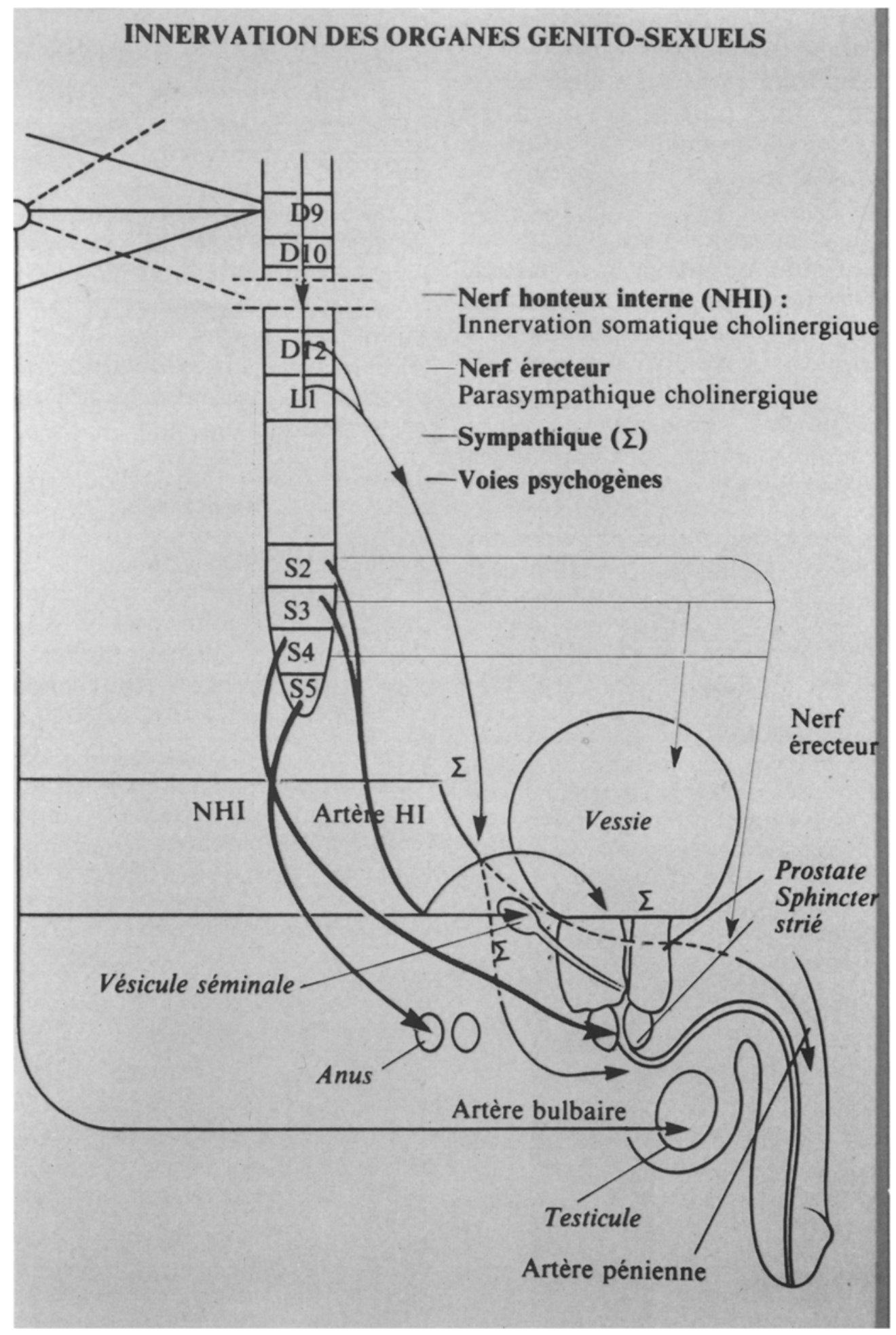

Figure 1: Anatomo-physiologie de l'érection. 
L'orgasme qui emprunte les voies spinothalamiques du plaisir et de la douleur n'est atteint que dans $20 \%$ des cas, alors que la douleur est ressentie chez $80 \%$ des paraplégiques.

Il faut dire aussi que cet orgasme est souvent anormal. En particulier chez le paraplégique haut, il se traduit souvent par des signes d'hyperréflectivité autonome : sueurs, céphalées, HTA. Parfois, ces signes n'apparaissent que lors des premiers rapports. Il est préférable que la vessie soit vidée avant le rapport. Les érections sont meilleures vessie pleine, mais la distention vésicale peut être responsable d'hyperréflexie ou de fuites mictionnelles lors du rapport (la masturbation étant parfois une méthode de vidange de certaines vessies réflexes).

Les paraplégiques bas ressentent le moins l'orgasme. L'éjaculation chez eux est très longue à obtenir.

L'influence de l'élément psychologique, même dans les paraplégies complètes et définitives, nous est apparu il y a 20 ans.

Un jeune tétraplégique est venu nous consulter pour impuissance d'apparition brutale à la suite de son mariage. La tétraplégie était complète, spasmodique, bien stabilisée puisque datant de 3 ans. Les érections étaient réflexes, particulièrement longues et avaient fait "la joie de nombreuses jeunes filles". Pourtant, dès le mariage, l'impuissance avait été totale. Ce jeune tétraplégique a récupéré érection et éjaculation avec l'aide attentive de sa femme, grâce à une sexothérapie de type Masters.

Doit-on douter de la séméiologie ? Il ne faudrait alors jamais affirmer qu'une lésion est complète. Existe-t-il une voie extra-médullaire? Il s'agit en effet de la voie ortho-sympathique entraînant vasoconstriction et contraction de l'éponge caverneuse. On est en train de mettre en évidence un centre cortical inhibiteur de l'érection.

\section{CONDUITE A TENIR VIS A VIS DE LA SEXUALITE DU PARAPLEGIQUE}

Il y a 10 ans, nous abordions le problème sexuel dès le début de la rééducation urinaire, donc dès la fin du choc spinal.

Le niveau neurologique joue un rôle fondamental dans le comportement psychologique du paraplégique. Le choc spinal dure de quelques jours à quelques semaines. Sa disparition est marquée par la réapparition de l'activité médullaire sous-lésionnelle. Ainsi, on voit réapparaitre l'érection réflexe dans les lésions au-dessus de D12 vertébral. Cette érection réapparait d'autant plus vite que le niveau est haut. Chez certains tétraplégiques hauts, qui ont d'ailleurs présenté une érection réflexe lors de l'accident, elle réapparaît en quelques heures. Cette érection se produit au moindre attouchement, par les draps, lors de la toilette, au changement de la sonde, etc. Parfois même, les malades avouent n'avoir jamais eu d'érection aussi "belle" avant le traumatisme médullaire. Ces malades sont donc rassurés sur leur possibilité d'érection. Celle-ci fait même l'objet de longues conversations et occupe en grande partie l'esprit de ces malades qui restent immobilisés dans leur lit. Il n'y a aucun souci à se faire à propos de leur libido. Elle est facilement entretenue par leurs propos, leurs lectures, les films, etc. Par contre, cette attitude d'homme viril va avoir de fâcheuses conséquences sur leurs compagnons de chambre qui seront toujours en choc spinal ou qui présenteront une lésion basse.

L'angoisse va s'emparer d'eux, ils vont devenir des candidats à une impuissance psychologique, alors que physiologiquement, ils sont peut-être de ceux qui doivent récupérer leurs fonctions génitales.

Nous considérions qu'il y avait urgence à évoquer la fonction sexuelle avant la première permission. En effet, à la première permission, tous les paraplégiques sont des candidats à l'impuissance d'origine psychologique, même ceux qui ont, avec leur lésion haute et complète, des érections réflexes et durables. 
Nous avons souvent vu apparaître des impuissances au domicile conjugal, alors que l'érection était très satisfaisante à l'hôpital.

L'homme neurologique qui rentre chez lui n'est plus le même. Pour lui, la première permission au domicile est une véritable aventure. L'épouse découvre un homme différent. Souvent elle a quitté un sportif, elle retrouve un handicapé qu'il faut parfois aider comme un enfant. Il est donc nécessaire que le couple se redécouvre. Il est évidemment indispensable de voir cette épouse et de lui expliquer ce qu'elle doit faire et ne pas faire. A cet entretien, il apparaît parfois qu'il sera impossible de réinserer cet homme dans son foyer. Le problème n'est plus sexuel, mais conjugal et social. Il est absolument fondamental d'interdire au patient tout rapport sexuel, en lui expliquant qu'il doit subir une rééducation, dont la première partie consiste à redécouvrir sa partenaire et à combler le vide sentimental qu'a laissé l'hospitalisation. Il faut recréer l'amour entre les deux partenaires et éliminer toute pitié.

De nombreux problèmes techniques vont se poser à ce couple et il ne faut pas y ajouter celui du rapport sexuel. Cependant, il faut expliquer au patient qu'il doit répondre aux avances de sa partenaire et qu'il doit essayer de la faire "jouir", si elle le demande, en évitant le rapport sexuel. Il faut lui recommander de laisser faire sa partenaire si son désir est très important et si l'érection est obtenue. Le patient ne doit pas s'occuper de cette érection. Il doit conserver le décubitus dorsal. Si la partenaire désire le coït, elle doit introduire elle-même cette verge et garder la "maîtrise" du rapport. En effet, l'intromission en décubitus ventral pose de grands problèmes à l'homme spinal. L'absence de sensibilité, l'absence de motricité obligent souvent à des délais importants pendant lesquels l'érection disparaît. Ainsi commence l'impuissance d'origine psychologique.
L'absence de force du malade, qui doit supporter tout son poids à bout de bras, empêche le rapport de se prolonger, le limitant alors à la simple intromission, du fait de l'épuisement du sujet. La longue durée du rapport est un élément impératif si l'éjaculation veut être obtenue.

Dans la majorité des cas, lorsque le couple est très uni, le patient passe rapidement outre l'interdit médical. Le rapport est alors ressenti comme un succès, presque un exploit. Lorsque le rapport est un échec, le malade comprend mieux la nécessité d'aller progressivement et conduit alors sa vie sexuelle comme sa rééducation motrice, rassuré par l'interdit médical. Il n'y a donc aucune différence dans cette rééducation sexuelle avec celle pratiquée par Masters chez l'impuisant somatiquement normal.

Cette technique de Masters doit être appliquée avec encore plus de rigueur au paraplégique impuissant (lésions basses ou lésions hautes, flasques par atteinte vasculaire par exemple). Il est absolument nécessaire que la partenaire comprenne que l'homme paraplégique peut être encore un "objet" sexuel. Cette verge flasque appartient au jeu de la femme. Elle seule, peut obtenir une rigidité. Il faut donc que la recherche des zones érogènes soit poussée au maximum. Il est souvent assez difficile de faire comprendre que ces zones existent toujours, même si l'homme ne les ressent plus. Il faut donc insister dans les explications, parfois apprendre à une femme comment caresser une verge, un scrotum, des cuisses, des fesses, etc. C'est là que le film de sexothérapie peut intervenir.

Les résultats de cette sexothérapie ou de cette rééducation sont souvent rapides et toujours bons quand la coopération de la partenaire est acquise. Le problème principal reste donc celui du couple : absence d'amour, mépris, frigidité, vaginisme ou absence de partenaire.

Les couples nés après la lésion neurologique présentent exceptionnellement des problèmes sexuels. 
L'apparition des médications vasoactives a complètement changé cet état de fait. Pendant quelques années, nous n'avons vu que les complications de celles-ci, comme nous n'avons plus vu que les complications urinaires. Nous n'avons plus participé à la rééducation génito-urinaire car elle avait disparu.

Depuis 5 ans, notre protocole d'étude de la fonction recto-génito-urinaire, selon le niveau neurologique, nous a permis de reprendre contact avec ces paraplégiques en dehors de la notion d'urgence ou de complication.

La stimulation visuelle permet de déterminer les érections psychogènes. L'utilisation du garrot peut permettre de maintenir cette érection psychogène ou réflexe et même d'obtenir une éjaculation par augmentation de la pression et de la stimulation au niveau du gland. On a en effet pu constater la disparition brutale de l'érection par pincement du gland, expliquant certaines détumescences brutales en position intra-vaginale.

Ce même bilan pratiqué très précocément au cours de l'hospitalisation loin de la première sortie a aussi permis de tester les injections intracaverneuses et le vacuum, si bien que tout patient est au courant de toutes les aides thérapeutiques non invasives.

\section{LA PLACE DES AUTO-INJECTIONS DE MEDICATIONS VASOACTIVES [5]}

\section{1) Rappel des médications employées et de leur mécanisme d'action}

a) Le chlorhydrate de papavérine est un alcaloïde de l'opium connu depuis le 19 ème siècle. Il diminue le calcium intracellulaire par différents mécanismes enzymatiques, il bloque également les canaux calciques. In vitro, la papavérine relâche la musculature lisse artérielle et trabéculaire. Sa demi-vie plasmatique est d'une à deux heures et son métabolisme est hépatique. Son administration par voie intracaverneuse semble responsable d'une réaction inflammatoire liée à la molécule ellemême. b) Les alpha-bloquants sont employés car il existe, au sein du corps caverneux, des récepteurs alpha-adrénergiques. Les récepteurs alpha 1 sont fonctionnellement les plus importants dans la contraction de la fibre musculaire lisse caverneuse. Le blocage du tonus alpha adrénergique pénien serait la première étape indispensable de l'érection.

- La phénoxybenzamine produit un blocage irréversible non spécifique des récepteurs alpha-adrénergiques. Par voie intra-caverneuse, elle n'est plus guère employée.

- La phentolamine est un antagoniste compétitif des récepteurs adrénergiques alpha 1 et alpha 2. Par voie intra-caverneuse, elle est souvent associée à la papavérine.

- Le moxisylyte est un antagoniste compétitif des récepteurs alpha 1 adrénergiques post-synaptiques. Par voie intra-caverneuse, il a fait l'objet d'une récente mise sur le marché (ICAVEX ${ }^{\circledR}$ ).

- La prostaglandine E1 est normalement présente dans le corps caverneux humain. Cette substance inhibe la neurotransmission adrénergique et exercerait un effet myorelaxant direct. Un système enzymatique permet sa destruction dans le corps caverneux.

- Le monoxyde d'azote est un puissant relaxant musculaire lisse. Le NO dans le corps caverneux peut être libéré par la cellule endothéliale en réponse à un message nerveux ou directement par les terminaisons nerveuses.

- Le linsidomine (CORVASAL ${ }^{\circledR}$ ) administré par voie intra-caverneuse provoque une libération de monoxyde d'azote par un mécanisme non enzymatique donc endothélium indépendant.

- Le vasoactive polypeptide (VIP) et le calcitonine gene related peptide (CGRP) sont tous deux des neuromédiateurs non adrénergiques, non cholinergiques du système 
parasympathique. Administrés par voie intra-caverneuse, ils relâchent le muscle lisse en inhibant la libération de calcium intra-cellulaire.

L'efficacité relative de ces différentes substances est mal connue dans la mesure où on ne dispose pas aujourd'hui de critère d'évaluation précis tant de l'effet immédiat des injections intra-caverneuses que du résultat du traitement par celles-ci.

Au vu de l'ensemble de la littérature, on peut cependant distinguer deux groupes de drogues. D'une part, les drogues facilitatrices de l'érection avec par ordre croissant d'efficacité le VIP, le CGRP, les alpha-bloquants et le sin-1, d'autre part, le groupe des drogues érectogènes représentées par la papavérine et la prostaglandine E1. Cette dernière pouvant s'avérer efficace après un échec de la papavérine. La mode est au mélange de substances vaso-actives.

\section{La technique}

Tous les spécialistes de l'impuissance ont insisté sur la nécessité de tester l'efficacité pharmacologique en milieu médical et sur la nécessité d'un apprentissage, tout en sachant que le résultat obtenu au cabinet du médecin et au domicile peut être fonction de l'état psychologique du patient. On voit actuellement de plus en plus de patients qui n'ont pas eu cet apprentissage. L'ordonnance du médecin traitant suffit pour se procurer de la papavérine. La publicité faite à l'ICAVEX ${ }^{\text {Q }}$ et la notice explicative de la technique enlèvent toute arrière-pensée au médecin généraliste. Le développement des auto-injections intracaverneuses est donc obligatoire. Un problème médico-légal risque de se poser avec la papavérine, puisque seul l'ICAVEX $\$$ (moxisylyte) a actuellement l'AMM. Il pourrait en effet être fait obligation au médecin de prouver que le moxisylyte est inefficace avant de prescrire un autre produit, même disponible en pharmacie. En cas d'incident (priapisme, nodule, hématome, douleurs ou autres), sa pleine respon- sabilité serait engagée, d'autant plus qu'il n'y aurait pas eu d'apprentissage, d'explications, en particulier des risques encourus.

Certains spécialistes ont un agrément ministériel, le plus souvent nominatif pour certains produits, dont la distribution n'est pas assurée en France. Seuls l'UROALPHA ${ }^{\circledR}$ injectable (moxisylyte) et le CORVASAL ${ }^{\circledR}$ sont disponibles dans les pharmacies des hôpitaux. La PROSTINE VR (prostaglandine E1) n'est plus délivrée en France par les pharmacies. Une expertise internationale concernant la prostaglandine E1 vient d'être réalisée, ce qui laisse supposer que ce produit pourrait avoir une AMM en France dans un avenir proche.

Les habitudes des prescripteurs sont très variables : certains utilisent encore le garrot, parfois une simple compression à la base de la verge. La majorité fait utiliser la technique la plus simple possible : verge tirée par la peau de façon à faire ressortir le corps caverneux, ponction latérale perpendiculaire au corps caverneux peau tendue, entre le gland et le pubis, avec une petite aiguille, au maximum 25 gauge, avec une seringue classique ou à insuline ou à l'autoinjecteur, qui éviterait le stress de la piqûre. Les produits sont assez stables, sauf le moxisylyte, qui ne peut pas être préparé plus de 6 heures avant l'injection.

\section{La morbidité}

La principale complication est représentée par le priapisme, celui-ci a surtout été observé après administration de papavérine. Il est beaucoup plus rare avec les alphabloquants et la prostaglandine E1. Diverses séries rapportent des pourcentages variables de priapisme après papavérine. Cette complication ne semble plus représenter un réel problème dans la mesure où les patients sont bien éduqués et où la dose de substance administrée a été préalablement déterminée lors de plusieurs injections. L'administration d'une dose faible initiale semble être volontiers réalisée. 
Le priapisme chez les patients neurologiques est plus fréquent, il existerait en effet un effet dose variable et une efficacité parfois intermittente de la substance administrée. Certains soulignent l'intérêt d'une fiche portée par le patient informant le médecin traitant de la possibilité du priapisme. Il est très important de faire comprendre à ces patients neurologiques ce qu'est un priapisme. En effet, les troubles de la sensibilité les empêchent de s'inquiéter de la présence d'un priapisme à haut débit, très dangereux puisque déterminant une hypoxie à partir de la $2^{\text {ème }}$ heure et une destruction du corps caverneux en 6 heures. Autant un individu normal ne supportera pas la douleur d'un tel priapisme, autant le patient neurologique endormi est capable de ne même pas se rendre compte qu'il est en priapisme. Il est donc préférable chez les neurologiques de provoquer en milieu médical un priapisme dont ils vont subir mais aussi voir le traitement par ponction. Certains patients traitent ainsi eux-mêmes, sans aucune conséquence locale, leur priapisme récidivant.

Les priapismes à bas débit, qui sont en fait des érections prolongées non douloureuses, élastiques non rigides, peuvent disparaître spontanément.

Certains utilisent des alpha-stimulants intra-caverneux type EPHEDRINE @ ou MIDODRIN ${ }^{\circledR}$. La noradrénaline et ses dérivés ne doivent plus être utilisés, du fait des risques d'HTA paroxystique. La fibrose musculaire est diversement appréciée selon les auteurs. Elle peut apparaitre après la première injection. Elle serait moins importante avec l'auto-injecteur qu'avec la seringue (Virag $0,4 \%$ contre $14 \%$ ), alors que l'injection manuelle permet de déplacer l'aiguille si le produit pénètre mal, ce qui est une cause de nodule. Les hématomes seraient aussi moins fréquents avec l'autoinjecteur. Le massage au point de ponction diminuerait le risque de nodule. La douleur à l'injection n'existe pas chez le paraplégique complet [12].
Le paraplégique utilise rarement le vacuum [8]. Il n'y a pratiquement plus d'indication de pose de prothèse pénienne chez le neurologique, en dehors des problèmes de rétraction de verge. L'implant pénien souple maintient une longueur suffisante de verge pour adapter un condom ou autre appareil à incontinence sans faire courir trop de risque d'ulcération ou de perforation du gland ou de la verge. Le stimulateur électronique implanté sur les racines sacrées sont au début de leur expérience. La lésion neurologique doit être complète. Les racines sensitives sont sectionnées. Ces stimulateurs de l'érection n'ont jamais été implantés pour ce seul but. Il s'agit toujours d'une indication de stimulateur pour la contraction vésicale auquel on ajoute un programme particulier pour l'érection. BRINDLEY a également implanté des stimulateurs pour l'éjaculation mais ses cas n'ont pas encore été publiés.

\section{LES PARAPLEGIES INCOMPLETES}

Tout paraplégique incomplet va réagir aussi bien que le paraplégique complet aux injections intra-caverneuses. Les problèmes psychologiques sont souvent plus complexes, surtout lorsque l'individu marche normalement.

Autant le paraplégique qui a accepté le fauteuil roulant et donc accepté le statut d'handicapé, trouve normal d'avoir dans une poche une sonde et dans l'autre une seringue, autant celui qui marche se prend toujours pour un homme normal.

\section{Le syndrome de la queue de cheval} traumatique ou non est celui qui a le plus de difficulté à accepter un statut "d'homme neurologique", pourtant il a échappé au fauteuil. Il a beaucoup de difficulté à accepter les autosondages et les auto-injections. Il va abandonner une fois sur deux celles-ci et souvent voir disparaître leur efficacité avec le temps. Il est volontiers candidat à la prothèse pénienne, comme il l'est au sphincter artificiel après incontinentation. 
2. Le spina bifida a en général remarquablement accepté son handicap et a trouvé seul son équilibre sexuel avec des érections qui ne sont pas toujours bonnes, mais dont il se contente, des éjaculations normales antérogrades selon ses dires, malgré l'incontinence urinaire et le col vésical béant. Il est d'ailleurs assez surprenant de voir des couples mariés depuis longtemps, venir pour des problèmes de stérilité avec une vie sexuelle normale, alors qu'il existe une incontinence urinaire et fécale. Bien sûr l'épouse était au courant de la situation avant le mariage. Cet état d'hygiène précaire n'a pas perturbé leur sexualité. Même si elle est intelligente, elle ne veut pas qu'on s'occupe des problèmes urinaires et rectaux de son mari, s'il estime être bien équilibré.

3. La sclérose en plaques est une maladie terrible, dégradante, car, outre le handicap moteur, il existe souvent, au cours de l'évolution, des troubles cérébelleux et intellectuels. La maladie va frapper un couple établi, normal. La dégradation physique et intellectuelle sera progressive, parfaitement perceptible par le patient et sa femme.

Les problèmes sont quotidiens, souvent urinaires, fécaux et n'encouragent pas la libido. Très souvent le dialogue est interrompu au niveau du couple. La répétition des échecs sexuels aggrave encore la situation. Le processus de séduction a disparu. Certains couples ont du mal à échanger leurs sentiments et leurs inquiétudes. Il arrive que des partenaires aillent jusqu'à se dissimuler des sentiments jugés inacceptables. Certains sclérosés en plaques se sentent coupables des conséquences de la maladie sur la relation conjugale. Ils ont le sentiment d'en demander beaucoup à leur conjoint. Au contraire, quelques fois, c'est la colère, la frustration ou la dépression qui domine la situation.

Au total, les bouleversements physiques et émotifs ont des répercussions sexuelles plus importantes que la lésion neurologique ellemême. D'ailleurs, on a noté que l'impuissance atteignait déjà 30 à $50 \%$ des patients dont la lésion neurologique était minime et sans relation avec cette impuissance [2].

On a démontré que, malgré l'insensibilité du pénis, la pléthysmographie nocturne pouvait être normale. On peut alors en conclure que l'élément psychologique est prépondérant [7]. Dans cette série, l'orgasme était toujours présent, mais à une moindre fréquence. On retrouvait $52 \%$ de problèmes sexuels, alors qu'il n'y en avait aucun avant le début de la maladie. Les symptômes étaient apparus brusquement [2].

Il faut rétablir le dialogue, personne ne peut engager un dialogue entre les conjoints à leur place. Il est peut-être nécessaire de donner certaines lignes directrices pour éviter qu'une discussion amicale ne dégénère en un débat stérile et blessant. Il faut demander aux sclérosés en plaques de susciter un climat d'ouverture dans le dialogue, en ne prenant pas de ton accusateur, ni moralisateur, ni carrément insistant, d'accepter toute proposition, par exemple le changement d'expérience sexuelle, sans penser qu'il s'agit de vice, de ne jamais discuter sur le coup de la colère, de la fatigue.

La réponse sexuelle peut être perturbée du fait des troubles de la sensibilité ou des troubles moteurs. Il est alors parfois nécessaire de trouver uniquement une solution de remplacement. Le dialogue dans le couple a dû permettre de mettre en évidence les changements de sensibilité, par exemple la douleur provoquée par la caresse. Il faut alors trouver une autre zone érogène. Il faut éliminer le défi de la performance quand l'érection est devenue rare et imprévisible. Le patient doit alors minimiser sa frustration et il faut éviter qu'il se replie sur lui même en évitant les contacts sexuels. La situation sera en effet très rapidement irréversible. C'est la même chose pour la femme, dont le coït est devenu désagréable à cause de contractures ou dont la perte de sensibilité a réduit la jouissance. Il est parfois nécessaire de faire des choix sexuels. La sexualité ne signifie pas obliga- 
toirement génitalité. La vie sexuelle n'est pas synonyme de coït.

Certains couples peuvent donc trouver, dans les activités sexuelles traditionnellement appelées préliminaires "une excitation physique et psychologique plus grande". Il est donc parfois nécessaire de faire une véritable éducation sexuelle à certains couples. Cette éducation peut être difficile, car l'apprentissage de l'érotisme peut être pris pour de la pornographie. Il n'est pas toujours facile de présenter des techniques de caresses, d'étreintes, de massages ou autres formes de toucher, par exemple la stimulation orale qui ne demande pas autant de mobilité que le coït, ou bien l'utilisation de vibrateurs ou dispositifs sexuels qui peuvent procurer une stimulation sexuelle avec un minimum d'effort. Les films doivent avoir là une grande importance. Il faut savoir s'accomoder des postures, en particulier lorsqu'il existe des spasmes en triple flexion, en utilisant le coït en position latérale et postérieure. Il faut savoir utiliser les myorelaxants à certaines heures, de façon à ce que leur activité maximale coincide avec l'activité sexuelle.

Il est parfois nécessaire de planifier l'activité sexuelle car la fatigue risque de réduire l'attrait sexuel, la fréquence des contacts et le plaisir éprouvé. La quantité d'énergie requise pour répondre à d'autres besoins peut inciter à laisser tomber la sexualité. Pour la plupart des gens, l'augmentation de la stimulation psychologique renforce l'érotisme. Il est parfois nécessaire d'utiliser des lectures ou des films érotiques ou certaines musiques etc... Ces formes de stimulation psychologique peuvent être bénéfiques chez ceux chez qui la sensibilité génitale est réduite. Il est par contre nécessaire qu'elle ne soit pas mal interprétée par le ou la partenaire, qui peut se demander pourquoi il ne constitue pas un stimulus suffisant.

Il est également nécessaire de veiller à l'hygiène génitale et en particulier au contrôle des sphincters. Comme l'orgasme s'accompagne de contractions du plancher pelvien, il peut y avoir "méprise neurologique". Les spasmes du sphincter strié habituels pourront être suivis de relaxation, d'où l'incontinence possible urinaire ou fécale. Il est donc nécessaire, dans certains cas, d'assurer une vidange vésicale et rectale. Au besoin, il faut utiliser le cathétérisme. Il est également possible de limiter l'absorption de liquides pendant les heures précédant l'activité sexuelle. Celle-ci peut être le prélude à un souper à la chandelle, plutôt que l'épilogue. Quand il existe une sonde à demeure, il est préférable de l'ôter. Certains patients conservent la sonde, en repliant le tube sur le pénis et en mettant un condom bien lubrifié. L'infection urinaire pose souvent des problèmes aux couples. Si l'hygiène est correcte, il n'y a pas de raison que cette infection se transmette au partenaire. Il faut insister sur la miction avant et après le rapport, au besoin le cathétérisme complémentaire, sur la toilette vaginale après le rapport. On a pu constater que certaines douches ou certains bains avant le rapport, en particulier avec la stimulation due au savonnage, amélioraient considérablement la stimulation sexuelle. Certains patients présentent des érections uniquement par ce moyen.

Les injections intra-caverneuses seront le dernier recours, mais il n'est pas rare d'obtenir une érection réflexe de bonne qualité au simple nettoyage de la verge avant l'injection, ce qui est bien la preuve que le travail de sexothérapie du couple n'a pas été fait. Le vacuum est plus souvent mieux accepté par l'épouse, mais il faut se méfier de l'absence de sensibilité, si on ne veut pas risquer écchymoses ou hématomes.

Finalement, il existe un peu plus d'indication de prothèses péniennes du fait de la présence des troubles des membres supérieurs : défaut de sensibilité, tremblement, troubles cérébelleux, etc... empêchant les manipulations les plus simples.

\section{LE PROBLEME DE LA LIBIDO}

Il existe souvent des troubles de la libido. Nous avons trouvé $20 \%$ de testostéronémie 
effondrée, même chez des sujets très jeunes. Il y avait toujours corrélation avec une diminution ou une disparition de la libido. La testostéronémie a vu multiplié son taux par 10 après injection de gonadotrophine et la libido est réapparue, comme si le testicule se mettait au repos dès qu'il n'y a plus d'activité sexuelle et de stimulation de la libido [9]. On comprend que, pendant toute la période de choc spinal, le paraplégique ait d'autres pensées que sexuelles.

Ce même état peut se présenter chez le paraplégique incomplet, traumatique ou non.

En fonction de l'âge, le testicule ne sera pas toujours sensible aux gonadotrophines. Il faut alors avoir recours au traitement androgénique de stimulation en particulier devant l'échec d'intra-caverneuse, bien sûr en surveillant la prostate.

Il reste cependant un deuxième cas de figure contre lequel il est difficile d'être efficace. L'individu, en général un paraplégique incomplet qui remarche, ne veut plus entendre parler de sexe car il ne sent rien. Il veut qu'on lui redonne sa sensibilité. Il n'a que faire de sa partenaire, de lui faire plaisir et même pour lui de retrouver un certain plaisir. Son discours est tranché, opératoire, comme celui, qui, condamné au fauteuil roulant, n'a qu'un objectif : jouer au foot. En général, la psychothérapie n'est pas efficace. Pour vivre bien paraplégique, il faut être intelligent.

\section{LA PHARMACOLOGIE DE L'ERECTION}

Il n'y a guère d'indication de traitement pharmacologique. Les anti-dépresseurs peuvent être indiqués, car il est obligatoire que le paraplégique fasse son deuil de sa vie antérieure.

Il est d'ailleurs préférable qu'il passe du stade de malade à celui d'handicapé avant sa sortie de l'hôpital.

Les anti-cholinergiques ne sont plus contreindiqués, puisqu'ils vont être utilisés sou- vent tôt pour faire disparaitre les contractions non inhibées de la vessie, afin d'obtenir une continence urinaire. Les médications vaso-actives seront suffisamment efficaces localement pour supprimer l'effet anérectile des anticholinergiques.

Les alpha-bloquants per os n'ont plus d'intérêt devant l'efficacité des intra-caverneuses et du fait du risque d'incontinence urinaire puisqu'ils relâchent col vésical et urèthre.

Nous avions retrouvé une hyper-prolactinémie relative dans notre population de paraplégique (12\% contre $10 \%$ [9]). Mais les anti-prolactinémiants n'ont pas révélé une véritable efficacité.

\section{CONCLUSION}

Le traitement actuel de l'impuissance du paraplégique passe quasi obligatoirement par les injections intra-caverneuses. On peut aussi considérer que tout paraplégique, quels que soient son niveau neurologique et son étiologie, peut récupérer une érection. L'information et l'apprentissage permettent au patient de gérer lui-même les complications, dont la plus grave et la plus fréquente reste le priapisme.

Les bilans classiques andro-sexologiques apportent cependant une meilleure utilisation de ces auto-injections, en apprenant à utiliser les voies réflexes et psychogènes, à utiliser différentes techniques (préludes, positions, anneau de contention etc...). Il y a peu d'indication de vacuum ou de prothèses péniennes. Il faut parfois faire appel, en cas de mauvaise libido, à des traitements complémentaires anti-dépresseurs ou de stimulation ou de substitution.

\section{REFERENCES}

1. BRINDLEY G.S. : Cavernosal alpha-blockade : a new technique for investigating and treating erectile impotence. In : But. J. Psychiatry, 1983, 143 : 332-337.

2. BURNFIELD P. : Sexual problems and multiple scleroses. In : Sexuality and Disability, 1980, 3 : 223-229. 
3. COURTOIS F., LERICHE A. : L'érection psychogène chez l'homme paraplégique. In : Ann. Réadaptation Méd. Phys., 1992, $35: 39-45$.

4. LERICHE A. : La rééducation sexuelle de l'homme paraplégique. In : Cah. Méd., 1979, 4, 20 : 13631371.

5. LERICHE A. : Les autoinjections de médications vasoactives, quelles leçons après 10 ans d'expérience ? In : Dialogue en Urologie, 1993, $10: 1-4$.

6. LERICHE A. : Pharmacologie intracaverneuse. In : Méd et Hyg, 1988, $46: 3408-3417$.

7. LERICHE A. : Sexualité et sclérose en plaques. In : Sclérose en plaques et médecine de rééducation, coll. Problèmes en Médecine de Rééducation, 1987, $\mathrm{n}^{\circ} 9: 90-101$.

8. LERICHE A. : Traitement palliatif de l'impuissance : injections intra-caverneuses au vacuum ? In : Andrologie, 1991, $1: 120$.

9. LERICHE A., BERARD E., VAUZELLE J.L. et al. : Histological and hormonal testiculous changes in spinal cord patients. In : Paraplegia, 1978, 15 : 274-279.

10. LUE T.F. : Intracavernous drug admnistration : its role in diagnosis and treatment of impotence. In : Sem. Urol, 1990, $8: 100$.

11. VIRAG R. : Intracavernous injection of papaverine for erectile failure In : Lancet 1982, 2 : 938.

12. VIRAG R., NOLLET F. : Auto-injections de médications vasoactives. Le bilan de 10 ans d'expérience. In : Dialogue en Urologie, 1993, $10: 4-6$.

\section{ABSTRACT \\ Treatment of the paraplegics' impotency}

\author{
Albert LeRICHE \\ Hospices Civils de Lyon, Hôpital Henry \\ Gabrielle, Unité d'Urologie, Route de \\ Vourles, BP 57, 69565 Saint-Genis \\ Laval Cedex
}

The author is summing up his 25 years experience in the treatment of paraplegics impotency to account for the present and almost continuous resulting use of self injections of vasoactive drugs.

The sexual reeducation, based on the neurological level, that is to say on the physiopathology, has disappeared for 10 years, as the urinary reeducation has been replaced by self catheterism. Nevertheless, there are some education rules left for the paraplegics and the couple in the relationship as well as in the technique.

Trying to obtain orgasm for the paraplegics has become a priority, since the erection is obtained easily through drugs.

A reference is made to the vasoactive drugs that have been used and their effects.

The behaviour is precise somewhat different in the case of the traumatic and total paraplegics as compared to the partial medical paraplegics (cauda equina, spina bifida, multiple sclerosis...) for whom psychology is different.

The sensitivity deficiencies which are the same for all of them mustn't make us forget that there are still reflex erogeneous spots that must be searched for.

Key-words : Paraplegic, impotency, vaso-active drugs. 